University of Nebraska - Lincoln

DigitalCommons@University of Nebraska - Lincoln

January 2002

\title{
White-Light-Induced Fragmentation of Toluene
}

A. M. Müller

Max-Planck-Institut für Quantenoptik, Hans-Kopfermann-Strasse 1, 85748 Garching, Germany

B. Witzel

Department of Molecular and Optical Physics, Albert-Ludwigs-Universität Freiburg, Hermann-HerderStrasse 3, 79104 Freiburg, Germany

Cornelis J. Uiterwaal

University of Nebraska - Lincoln, cuiterwaal2@unl.edu

J. Wanner

Max-Planck-Institut für Quantenoptik, Hans-Kopfermann-Strasse 1, 85748 Garching, Germany

K.-L. Kompa

Max-Planck-Institut für Quantenoptik, Hans-Kopfermann-Strasse 1, 85748 Garching, Germany

Follow this and additional works at: https://digitalcommons.unl.edu/physicsuiterwaal

Part of the Physics Commons

Müller, A. M.; Witzel, B.; Uiterwaal, Cornelis J.; Wanner, J.; and Kompa, K.-L., "White-Light-Induced Fragmentation of Toluene" (2002). C.J.G.J. Uiterwaal Publications. 12.

https://digitalcommons.unl.edu/physicsuiterwaal/12

This Article is brought to you for free and open access by the Research Papers in Physics and Astronomy at DigitalCommons@University of Nebraska - Lincoln. It has been accepted for inclusion in C.J.G.J. Uiterwaal Publications by an authorized administrator of DigitalCommons@University of Nebraska - Lincoln. 


\title{
White-Light-Induced Fragmentation of Toluene
}

\author{
$\underline{\text { A. M. Müller }}^{1}{ }^{*}, \underline{\text { B. Witzel }}^{2}{ }^{\dagger}, \underline{\text { C. J. G. J. Uiterwaal }}{ }^{1 \neq \S}, \underline{\text { J. Wanner }}^{1}$, and $\underline{\text { K.-L. }}$ \\ Kompa $^{1}$ \\ ${ }^{1}$ Max-Planck-Institut für Quantenoptik, Hans-Kopfermann-Strasse 1, 85748 \\ Garching, Germany \\ ${ }^{2}$ Department of Molecular and Optical Physics, Albert-Ludwigs-Universität \\ Freiburg, Hermann-Herder-Strasse 3, 79104 Freiburg, Germany
}

Received 15 August 2001; published 21 December 2001

We investigate the influence of continuum generation on the photofragmentation dynamics of the toluene molecule. By sending an 80-fs Ti:sapphire laser beam through a 2-mm-thick $\mathrm{CaF}_{2}$ plate we show that even moderately broadened laser frequency spectra considerably increase the degree of fragmentation, while it is nearly absent without continuum generation. The measured broadening of the frequency spectra and the known Raman-active vibrational modes of toluene afford an explanation in terms of a stimulated Raman scattering scheme, in which the molecule absorbs large amounts of vibrational energy.

(C)2002 The American Physical Society

URL: http://link.aps.org/abstract/PRL/v88/e023001

DOI: 10.1103/PhysRevLett.88.023001

PACS: 33.80.-b, 42.65.Dr

\footnotetext{
* Present address: JILA, University of Colorado and National Institute of Standards and Technology, Boulder, CO 80309-0440.

† To whom correspondence should be addressed.Email addresses: witzel@unifreiburg.de; cuiterwaal2@unlnotes.unl.edu \#

${ }^{\S}$ Present address: Department of Physics and Astronomy, University of NebraskaLincoln, 116 Brace Lab, Lincoln, NE 68588-0111.
} 


\title{
White-Light-Induced Fragmentation of Toluene
}

\author{
A. M. Müller, ${ }^{1, *}$ B. Witzel, ${ }^{2, \dagger}$ C. J. G. J. Uiterwaal, ${ }^{1, \dagger},{ }^{\ddagger}$ J. Wanner, ${ }^{1}$ and K.-L. Kompa ${ }^{1}$ \\ ${ }^{1}$ Max-Planck-Institut für Quantenoptik, Hans-Kopfermann-Strasse 1, 85748 Garching, Germany \\ ${ }^{2}$ Department of Molecular and Optical Physics, Albert-Ludwigs-Universität Freiburg, Hermann-Herder-Strasse 3, \\ 79104 Freiburg, Germany
}

(Received 15 August 2001; published 21 December 2001)

\begin{abstract}
We investigate the influence of continuum generation on the photofragmentation dynamics of the toluene molecule. By sending an 80-fs Ti:sapphire laser beam through a 2-mm-thick $\mathrm{CaF}_{2}$ plate we show that even moderately broadened laser frequency spectra considerably increase the degree of fragmentation, while it is nearly absent without continuum generation. The measured broadening of the frequency spectra and the known Raman-active vibrational modes of toluene afford an explanation in terms of a stimulated Raman scattering scheme, in which the molecule absorbs large amounts of vibrational energy.
\end{abstract}

DOI: $10.1103 /$ PhysRevLett.88.023001

Introduction.-The study of white-light production induced by the interaction of an ultraintense laser pulse with condensed optical transparent media has been the subject of several recent investigations [1,2]. Applications such as optical parametric amplifiers [3] for production of tunable ultrafast light pulses [4] or the use of glass-fiber techniques [5,6] for ultrashort pulse generation are now available. Particularly this progress allows implementation and development of coherent control techniques, which are important for manipulation of molecular dissociation paths in laser-molecule ultrashort pulse interaction. The white-light continuum can also be used for time-resolved broadband absorption and excitation spectroscopy [7], as well as for dynamic characterization of laser-induced structural transitions. The underlying process of femtosecond continuum generation, most probably generated by self-phase modulation $[8,9]$, is far from being completely understood. It could be shown that the circumstances for observing white-light production are strongly related to a critical laser power threshold [10]

$$
P_{\mathrm{cr}}=\frac{c \varepsilon_{0} \lambda^{2}}{8 \pi n_{2}}
$$

where $c$ and $\lambda$ are, respectively, the speed of light and the wavelength of the laser light (both in vacuo); $n_{2}$ is the nonlinear index of refraction, defined here as $n=n_{0}+n_{2} E^{2}$ $\left(n_{0}\right.$ : linear refractive index; $E$ : peak electric field strength), not to be confused with the alternative intensity-based definition of this quantity given by $n=n_{0}+\gamma I$. Lasers commonly used in intense-field photoionization experiments have powers of several GW, which is far beyond the critical power of optical materials commonly used. $\mathrm{CaF}_{2}$, for instance, has $P_{\mathrm{cr}} \sim 2.7 \mathrm{MW}$. Although any laser beam with a power exceeding this critical value could produce a white-light continuum in principle, the extent to which this happens strongly depends on the optical path length in the nonlinear medium and the intensity of the beam. A good measure of the extent of self-focusing (and, hence, of continuum generation) taking place when a laser
PACS numbers: 33.80.-b, 42.65.Dr

beam travels over a distance $L$ in the $z$ direction through a medium with intensity-based nonlinear index $\gamma$ is given by the $B$ integral, defined as [11]

$$
B=\frac{2 \pi}{\lambda} \int_{0}^{L} \gamma I(z) d z .
$$

When this dimensionless number stays below a limit of 3 to 5 [11] self-focusing effects are negligible. An equivalent measure is the number $Q$, defined as [9] $\gamma I L / c \tau$ $(\tau=$ pulse duration; $I=$ intensity). The present experiments are conducted under conditions where continuum generation starts to become noticeable as modulation and a moderate broadening of the frequency spectrum. As a consequence, we observe a marked influence on the ionization and dissociation dynamics of the toluene molecule. This molecule was chosen as an example; the basic nature of the induced behavior is general and is likely to play an important role in nearly all studies of molecular dissociation processes.

The experimental setup is reported in previous work [12]. A Ti:sapphire laser system (Spectra Physics) is used, delivering $80-\mathrm{fs}$, $800-\mathrm{nm}$ pulses at a repetition rate of $1 \mathrm{kHz}$ with a maximum energy of $2.0 \mathrm{~mJ} /$ pulse (shot-toshot stability $\pm 4 \%$ ). The experimental studies reported are performed at the fundamental wavelength. The unfocused laser beam has a Gaussian spatial profile with a FWHM diameter of $(5.37 \pm 0.02) \mathrm{mm}$. Assuming the temporal shape of the pulse to be Gaussian, we determine a FWHM of $(80 \pm 1)$ fs, by standard autocorrelation techniques. Hence, the peak intensity of the unfocused beam is $I_{0}=4.2 \times 10^{10} \mathrm{~W} / \mathrm{cm}^{2}$. The laser beam is focused in the first acceleration zone of a Wiley-McLaren-type time-of-flight (TOF) mass spectrometer [13]. Experiments are conducted with two different planoconvex, uncoated fused silica lenses with focal lengths of 300 and $1000 \mathrm{~mm}$. For beam diagnostic purposes a flipper mirror is mounted behind the lens, which allows redirection of the beam to a CCD camera system protected with a variable attenuator. In this way the spatial profile of the focus can be analyzed 
outside the TOF for each lens independently. We use a 2-mm-thick $\mathrm{CaF}_{2}$ laser flat as the entrance window of the spectrometer. This material was chosen because its large band gap of $10.2 \mathrm{eV}$ [2] makes it particularly suited to continuum generation. It should be noted that a multitude of optical transparent materials often used would lead to white-light production under our experimental conditions [1]. Depending on the focal length of the lens used, the laser intensity at the entrance window can be chosen in the range $\sim 2 \times 10^{10}$ to $\sim 3 \times 10^{11} \mathrm{~W} \mathrm{~cm}^{-2}$, corresponding to cumulative $B$-integral values between $\sim 0.7$ and $\sim 1.5$. We do not observe significant changes such as filamentation in the spatial beam profile when the laser energy is varied. A sector filter disk mounted in front of the lens enables attenuation of the beam by up to 2 orders of magnitude. This allows experiments with comparable laser intensities at focus but with different laser intensities at the entrance window. The frequency spectrum of the laser beam is recorded by using a fiber spectrometer (Ocean Optics SD2000) placed several meters behind the exit window (2-mm laser flat $\left.\mathrm{CaF}_{2}\right)$ and centered at the optical axis.

Each spectral measurement is an average over 1000 laser shots. Toluene vapor (Merck Uvasol, purity 99.9\%) is admitted to the vacuum chamber by means of a variable leak valve. Depending on the experimental requirements, the partial pressure was between $10^{-8}$ and $10^{-7}$ mbar. Under these conditions space charge effects or electron impact ionization of rest gases do not have any influence on the experiment.

Results.-First we demonstrate the influence of the increasing $B$-integral value on ionization and dissociation of toluene after short-pulse laser interaction. This experiment is performed with a 1000-mm lens. Mass spectra of toluene and the corresponding wavelength spectra are taken as a function of the intensity to which the $\mathrm{CaF}_{2}$ entrance window is exposed (Fig. 1). The ratio of the intensity at focus to that at the entrance window is $I_{f} / I_{w}=20$. The recorded wavelength spectra (Fig. 1a) are expected to be somewhat broader than those at focus, because the radiation passes through the exit window before it is analyzed by the fiber spectrometer. For better visualization the mass spectra of Figs. 1b and 1c are normalized to the parent ion mass peak (Fig. 1c), and the vertical scale for the low-mass part of the spectrum (Fig. 1b) is expanded by a factor of 100 . Clearly, increasing the intensity not only causes spectral broadening of the radiation (Fig. 1a) but also leads to increasing fragmentation (Fig. 1b). To demonstrate this more quantitatively, we show in Fig. 1d, as an example, the increase of the sum of the yields for masses $27\left(\mathrm{C}_{2} \mathrm{H}_{3}{ }^{+}\right)$, $39\left(\mathrm{C}_{3} \mathrm{H}_{3}{ }^{+}\right), 51\left(\mathrm{C}_{4} \mathrm{H}_{3}{ }^{+}\right)$, and $65\left(\mathrm{C}_{5} \mathrm{H}_{5}{ }^{+}\right)$for increasing $I_{w}$. Many more peaks can be identified in Fig. 1b; peak broadening and splitting due to kinetic energy deposition and doubly charged ions are observed as well.

In principle, the increased fragmentation observed in Fig. 1 could be due either to the increasing intensity or to the increasing radiation bandwidth. To shed further light on the fragmentation mechanism we have carried out an experiment with two different lenses: $f=300 \mathrm{~mm}$ (Fig. 2a) and $f=1000 \mathrm{~mm}$ (Fig. 2b). Although the mass spectrum of Fig. 2a is recorded with a higher focal intensity $\left(I_{f}=1 \times 10^{14} \mathrm{~W} \mathrm{~cm}^{-2}\right)$ than that of Fig. $2 \mathrm{~b}\left(I_{f}=\right.$ $6 \times 10^{13} \mathrm{~W} \mathrm{~cm}^{-2}$ ), strong fragmentation is observed only in the latter. Obviously, this is a result of the different

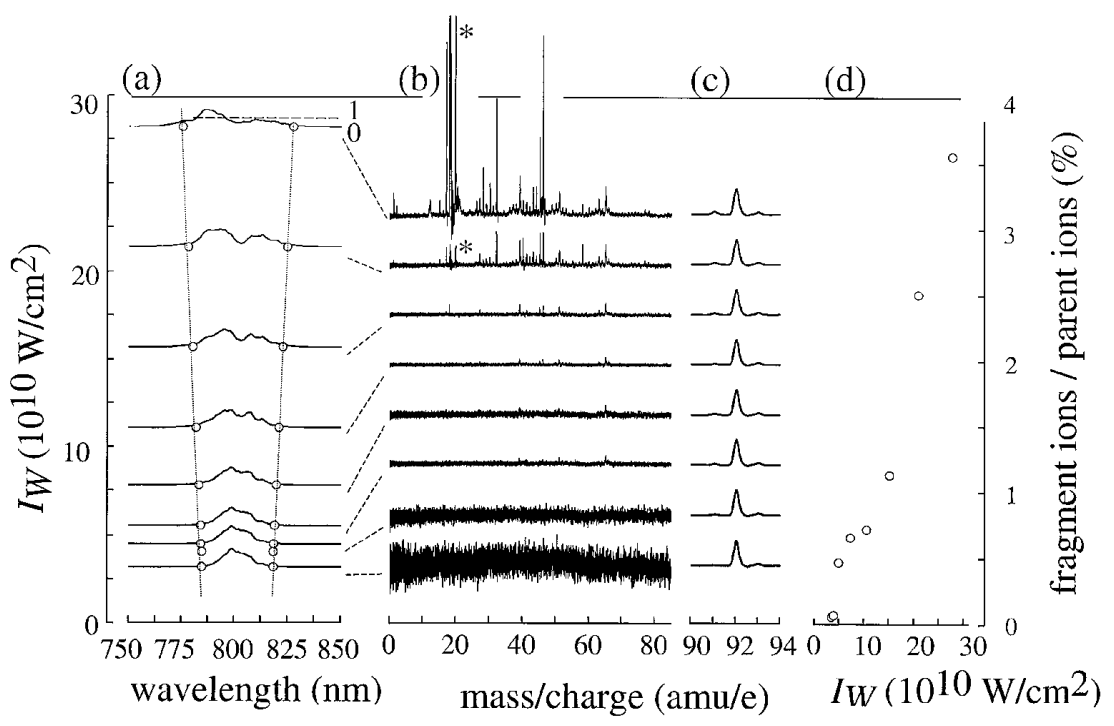

FIG. 1. Mass spectra of toluene and corresponding wavelength spectra of the laser light used, recorded at different intensities at the $\mathrm{CaF}_{2}$ entrance window $I_{w}$. Panel (a): wavelength spectra for various values of $I_{w}$. Blank circles and straight dashed lines indicate calculated Stokes and anti-Stokes cutoffs. Panel (b): mass spectra for the same $I_{w}$ values as in (a), normalized to the parent ion peak (c). The $\mathrm{H}_{2} \mathrm{O}^{+}$peak (marked with an asterisk) is clipped. The vertical scale for masses below 88 mass units is enlarged by a factor of 100. Panel (d): ratio of integrated fragment peaks [sum of masses $27\left(\mathrm{C}_{2} \mathrm{H}_{3}{ }^{+}\right), 39\left(\mathrm{C}_{3} \mathrm{H}_{3}{ }^{+}\right), 51\left(\mathrm{C}_{4} \mathrm{H}_{3}{ }^{+}\right)$, and $65\left(\mathrm{C}_{5} \mathrm{H}_{5}{ }^{+}\right)$] to integrated parent ion peak [panel (c)]. 


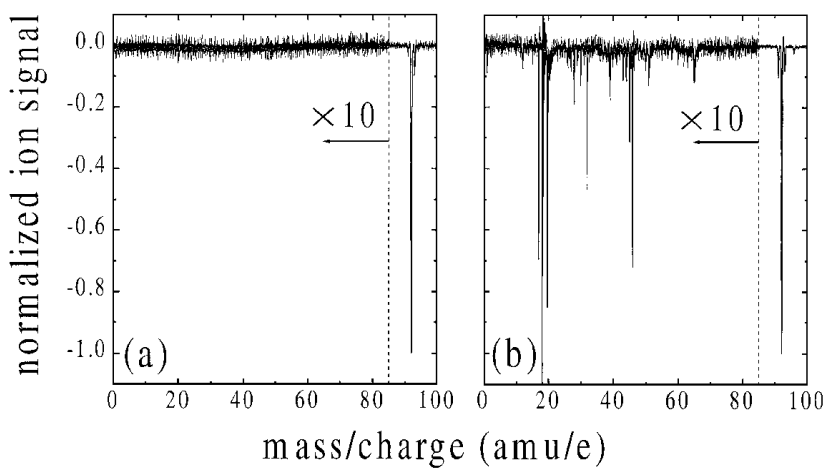

FIG. 2. Mass spectra of toluene. Panel (a): 300-mm focal length lens; $I_{w}=7 \times 10^{10} \mathrm{~W} \mathrm{~cm}^{-2}, I_{f}=1 \times 10^{14} \mathrm{~W} \mathrm{~cm}^{-2}$; panel (b): 1000 -mm focal length lens; $I_{w}=3 \times 10^{11} \mathrm{~W} \mathrm{~cm}^{-2}$, $I_{f}=6 \times 10^{13} \mathrm{~W} \mathrm{~cm}^{-2}$.

peak intensities to which the $\mathrm{CaF}_{2}$ entrance window is exposed $\left(I_{w}=7 \times 10^{10} \mathrm{~W} \mathrm{~cm}^{-2}\right.$ in Fig. $2 \mathrm{a}$ and $I_{w}=3 \times$ $10^{11} \mathrm{~W} \mathrm{~cm}^{-2}$ in Fig. 2b). We conclude that the increasing fragmentation as seen in Figs. $1 \mathrm{~b}$ and $1 \mathrm{~d}$ is the result of the increasing radiation bandwidth (Fig. 1a), and not of the increasing intensity.

Discussion.-One possible mechanism by which an increased bandwidth can lead to increased fragmentation is a stimulated Raman scattering process. In such a scenario a significant number of difference frequencies are absorbed by Raman-active modes of the neutral toluene molecule and/or of ionized toluene. To support this view, we calculated the availability of difference frequencies. Having measured the spectral content $S(\omega)$ of the nonlinearly distorted pulses (Fig. 1a), one can define the strength of a specific difference frequency $\omega^{\prime}$ as $D\left(\omega^{\prime}\right)=$ $\int S(\omega) S\left(\omega+\omega^{\prime}\right) d \omega$. In Fig. 3, such difference frequency spectra $D\left(\omega^{\prime}\right)$ are shown for various intensities $I_{w}$ at the entrance window. Clearly, the range of difference frequencies available is significantly broadened if $I_{w}$ is increased, a particularly strong increase occurring between 200 and $800 \mathrm{~cm}^{-1}$. Nine vibrational modes of the neutral toluene molecule exist in this range, eight of which are known to be Raman active [14]. Seven of these Ramanactive modes (vertical lines in Fig. 3) involve vibrations of the carbon backbone. The strong increase of $D\left(\omega^{\prime}\right)$ in this range can thus bring about strong vibrational excitation of the molecule, giving it enough internal energy to dissociate after ionization. Alternatively, part or all of the absorption of difference frequencies could take place in the ionized molecule. It is known that so-called soft ionization, i.e., photoionization of toluene with minimal fragmentation, takes place at $790 \mathrm{~nm}$ [15], $750 \mathrm{~nm} \mathrm{[16],}$ and $375 \mathrm{~nm} \mathrm{[15].} \mathrm{Therefore,} \mathrm{it} \mathrm{seems} \mathrm{improbable} \mathrm{that} \mathrm{any}$ of the frequencies created is responsible by itself for the increased fragmentation. Another possible mechanism could be stepwise multiphoton resonant ionization. This would imply the availability in sufficient quantities of as many frequencies as there are steps in such a process, including the frequency of the first electronic excitation

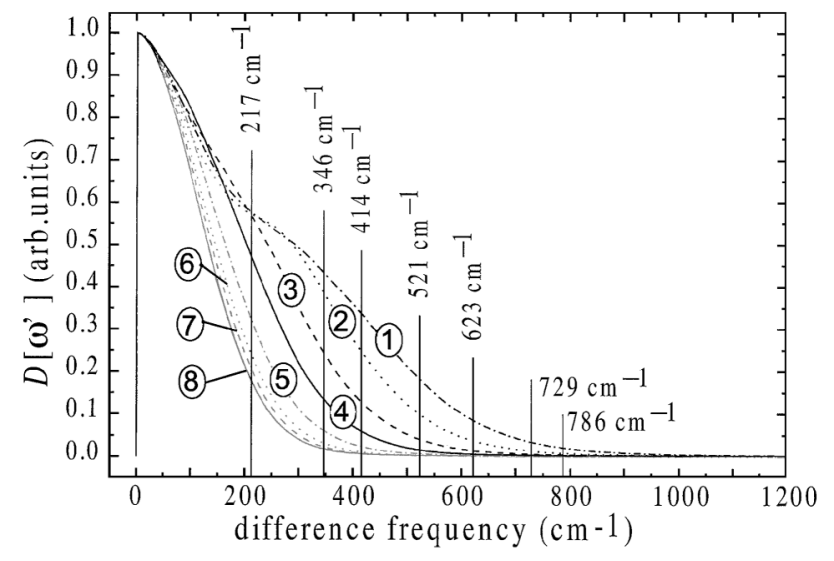

FIG. 3. Availability of difference frequencies as derived (see text) from the measured wavelength spectra recorded with specified intensities $I_{w}$ at the $\mathrm{CaF}_{2}$ entrance window (cf. Fig. 1a). [(1) $I_{w}=27.7 \times 10^{10} \mathrm{~W} \mathrm{~cm}^{-2}$, (2) $I_{w}=20.9 \times 10^{10} \mathrm{~W} \mathrm{~cm}^{-2}$, (3) $I_{w}=15.2 \times 10^{10} \mathrm{~W} \mathrm{~cm}^{-2}$, (4) $I_{w}=10.6 \times 10^{10} \mathrm{~W} \mathrm{~cm}^{-2}$, (5) $I_{w}=7.33 \times 10^{10} \mathrm{~W} \mathrm{~cm}^{-2} \mathrm{~d}$, (6) $I_{w}=5.04 \times 10^{10} \mathrm{~W} \mathrm{~cm}^{-2}$, (7) $I_{w}=3.99 \times 10^{10} \mathrm{~W} \mathrm{~cm}^{-2} \mathrm{~d}$, (8) $\left.I_{w}=3.55 \times 10^{10} \mathrm{~W} \mathrm{~cm}^{-2}\right]$. Vertical lines indicate Raman-active modes [14] of the carbon backbone of the neutral toluene molecule between 200 and $800 \mathrm{~cm}^{-1}$.

at $4.6 \mathrm{eV}$ [17]. [The photon energy for the fundamental wavelength $(800 \mathrm{~nm})$ of the Ti:sapphire laser is $1.55 \mathrm{eV}$.] Because this concept is resonant, one might argue that it could be driven by undetectably small VIS/UV/VUV components in the frequency spectra of Fig. 1a. To clarify this issue, we made calculations based on analytical and numerical models for nonlinear spectral broadening [1,9]. The Stokes and anti-Stokes cutoff frequencies we find are indicated as open circles in Fig. 1a and are in good agreement with experiment. Clearly, under our experimental conditions the anti-Stokes wing of the continuum does not even reach visible frequencies, so that a stepwise multiphoton resonant scheme can be ruled out. In our calculations, we find $Q \ll 1$ [9] ( $Q \sim 0.02)$, which confirms that there is only a very moderate degree of continuum generation with symmetric Stokes and anti-Stokes cutoff frequencies as shown in Fig. 1a.

Conclusion.-In conclusion, we have shown that moderate amounts of nonlinear spectral broadening may already lead to fundamental changes in the fragmentation behavior of a polyatomic molecule. An explanation is afforded by a stimulated Raman process allowing the molecule or the molecular ion to absorb large amounts of vibrational energy. The general nature of this phenomenon leads us to believe that it can be employed in coherent or optimal control of fragmentation.

B. W. is obliged to Max-Planck-Institut für Quantenoptik for the possibility of participating in this work and acknowledges DFG (Deutsche Forschungsgemeinschaft) for support. The authors also thank W. Fuß and H. Schröder for stimulating discussions. They acknowledge the technical help of W. Ritt and A. Steyer. 
*Present address: JILA, University of Colorado and National Institute of Standards and Technology, Boulder, CO 80309-0440.

${ }^{\dagger}$ To whom correspondence should be addressed. Email addresses: witzel@uni-freiburg.de; cuiterwaal2@ unlnotes.unl.edu

†Present address: Department of Physics and Astronomy, University of Nebraska-Lincoln, 116 Brace Lab, Lincoln, NE 68588-0111.

[1] A. Brodeur and S. L. Chin, J. Opt. Soc. Am. B 16, 637 (1999).

[2] A. Brodeur and S. L. Chin, Phys. Rev. Lett. 80, 4406 (1998).

[3] K. R. Wilson and V. V. Yakovlev, J. Opt. Soc. Am. B 14, 444 (1997).

[4] S. Cussat-Blanc, A. Ivanov, D. Lupinski, and E. Freysz, Appl. Phys. B 70, 247 (2000).

[5] A. Baltuska, Z. Wei, M. S. Pshenichnikov, D. A. Wiersma, and R. Szipocs, Appl. Phys. B 65, 175 (1997).

[6] R. L. Fork, C. V. Shank, C. Hirlimann, and R. Yen, Opt. Lett. 8, 1 (1983).

[7] The Supercontinuum Laser Source, edited by R. R. Alfano (Springer, New York, 1989).

[8] R. R. Alfano and S. L. Shapiro, Phys. Rev. Lett. 24, 584 (1970).
[9] G. Yang and Y. R. Shen, Opt. Lett. 9, 510 (1984).

[10] P. W. Milonni and J. H. Eberly, Lasers (Wiley, New York, 1988).

[11] A. E. Siegman, Lasers (University Science Books, Mill Valley, CA, 1986).

[12] A. M. Müller, C. J. G. J. Uiterwaal, B. Witzel, J. Wanner, and K.-L. Kompa, J. Chem. Phys. 112, 9289 (2000).

[13] W. C. Wiley and I. H. McLaren, Rev. Sci. Instrum. 26, 1150 (1955).

[14] L. M. Sverdlov, M. A. Kovnor, and E. P. Krainov, Vibrational Spectra of Polyatomic Molecules (Wiley, New York, 1974).

[15] D. J. Smith, K. W. D. Ledingham, R. P. Singhal, H. S. Kilic, T. McCanny, A. J. Langley, P. F. Taday, and C. Kosmidis, Rapid Commun. Mass Spectrom. 12, 813 (1998).

[16] K. W. D. Ledingham, D. J. Smith, R. P. Singhal, T. McCanny, P. Graham, H. S. Kilic, W. X. Peng, A. J. Langley, P. F. Taday, and C. Kosmidis, J. Phys. Chem. A 103, 2952 (1999).

[17] UV Atlas of Organic Compounds, edited by H. H. Perkampus, J. Sandeman, and C. J. Timmons (Butterworths, London, 1966), Vol. II. 